\title{
A closer look at Black-Scholes option thetas
}

\author{
Douglas R. Emery • Weiyu Guo • Tie Su
}

Published online: 11 October 2007

(C) Springer Science \& Business Media, LLC 2007

\begin{abstract}
This paper investigates Black-Scholes call and put option thetas, and derives upper and lower bounds for thetas as a function of underlying asset value. It is well known that the maximum time premium of an option occurs when the underlying asset value equals the exercise price. However, we show that the maximum option theta does not occur at that point, but instead occurs when the asset value is somewhat above the exercise price. We also show that option theta is not monotonic in any of the parameters in the Black-Scholes option-pricing model, including time to maturity. We further explain why the implications of these findings are important for trading and hedging strategies that are affected by the decay in an option's time premium.
\end{abstract}

Keywords Black-Scholes option pricing model - Option theta - Time decay

JEL Classifications $\mathrm{G} 10 \cdot \mathrm{G} 12$

\section{Introduction}

An option theta is the sensitivity of the option's price to changes in the option's time to maturity. It measures the rate at which the option's time premium decays over

D. R. Emery $\cdot$ T. Su $(\bowtie)$

Department of Finance, University of Miami, P.O. Box 248094, Coral Gables, FL 33124-6552, USA

e-mail: tie@miami.edu

D. R. Emery

e-mail: demery@miami.edu

W. Guo

Department of Finance, University of Nebraska-Omaha, 6001 Dodge Street, Omaha, NE 68182, USA e-mail:wguo@unomaha.edu 
time, as the option's remaining life dissipates. Option thetas are used in risk management, and can be used to measure the cost and benefit of an option hedge that depends on the natural decline in time to maturity.

This paper takes a closer look at Black-Scholes option thetas of European-style call and put options. The results answer two questions about these option thetas that have important implications for trading and hedging. First, at what underlying asset value (relative to the exercise price) does the option theta achieve its maximum? Or, equivalently, at what underlying asset value does an option's time premium have the fastest rate of dissipation over time? Second, are option thetas monotonic functions of the other parameter values, the underlying asset's return volatility, the option's time to maturity, and the risk-free interest rate? The answers to these questions can help option traders, hedgers, and speculators to better position their option trading strategies and offer them the possibility of managing the decay of option time premium to their benefit.

In the U.S., index options are extremely actively traded. Trading volume of U. S. index options is much higher than that of options on individual stocks. All index options, with the exception of options on the S\&P 100 OEX Index, are European style. While all options on individual stocks are American-style options, option theory tells us that if the underlying stock does not pay dividends before the expiry of the option, then the value of an American-style call option is the same as the value of an otherwise identical European-style call option. Given the fact that fewer than $22 \%$ of all stocks pay any dividend, and the fact the average dividend-paying stocks produce a dividend yield that is less than $1 \%$, we conclude that there is significant amount of trading in European-style and European-style like options, where the Black-Scholes option pricing model can be used to produce approximation of option prices.

The Black and Scholes (1973) option-pricing model was a seminal breakthrough in pricing derivatives. Numerous studies have examined the model's performance with respect to pricing. Black (1975), Emanuel and MacBeth (1982), MacBeth and Merville (1979), and Rubinstein (1985) all report that the Black-Scholes model tends to systematically misprice in-the-money and out-of-the-money options. However, the model is accurate enough that finance professionals routinely use extended and modified versions of the Black-Scholes model to value many types of options, including equity options.

Option thetas have attracted significant attention from both academia and practitioners. Discussions of option thetas can be found in almost all textbooks on derivatives and a number of academic papers. However, properties of option thetas have not been closely examined. Pelsser and Vorst (1994) and Chung and Shackleton (2002) use efficient numerical differentiation methods to compute option thetas. Chance (1994), focusing primarily on call options, partitions a call option into a margin value and an insurance policy, and discusses interpretations of option "Greeks." Naib (1996) studies the option effect, underlying effect, and curve effect in an option theta. Alexander and Stutzer (1996) present a graphical description of Black-Scholes put option thetas and time premiums. 
This paper is most closely related to Alexander and Stutzer (1996). We examine the properties of both call and put option thetas in the Black-Scholes model. We use the Black-Scholes option-pricing framework because of its wide acceptance, its simplicity and elegance, and its mathematical tractability. All conclusions and inferences derived in this paper can be used as benchmarks for other more sophisticated option-pricing models.

Our paper contributes to the options literature by focusing on five issues that have not been addressed by previous studies: (1) determining the upper and lower bounds on option thetas, (2) deriving the relation between the value of the underlying asset and option thetas, (3) deriving the relation between the underlying asset's return volatility and option thetas, (4) deriving the relation between the option's remaining time to maturity and option thetas, and (5) deriving the relation between the risk-free interest rate and option thetas. In addition, we examine the implications of our findings for trading and hedging with options.

The paper is organized as follows. The next section presents the Black and Scholes (1973) option-pricing model and the Black-Scholes option thetas for European-style call and put options. The subsequent five sections address each of the five issues about option thetas detailed above. The eighth section discusses how our results can be applied in hedging and trading option combinations. The final section concludes the paper. There are four attached appendices.

\section{Black-Scholes option thetas}

The Black-Scholes option-pricing model for European-style call and put options is given by the following standard notation:

$$
\begin{aligned}
& C=S_{0} N\left(d_{1}\right)-X e^{-r t} N\left(d_{2}\right) ; \\
& P=X e^{-r t} N\left(-d_{2}\right)-S_{0} N\left(-d_{1}\right)
\end{aligned}
$$

where $d_{1}=\frac{\ln \left(S_{0} / X\right)+\left(r+\sigma^{2} / 2\right) t}{\sigma \sqrt{t}}$, and $d_{2}=d_{1}-\sigma \sqrt{t}$;

$C$ and $P$ are the values of European-style call and put options, respectively;

$S_{0}$ is the current value of the underlying non-dividend paying asset;

$X$ is the option's exercise price;

$r$ is the annualized continuously compounded risk-free rate of interest;

$t$ is the option's time to maturity;

$\sigma$ is the standard deviation of the rate of return on the underlying asset;

$N(d)$ is the cumulative distribution function of the standard normal distribution, whose probability density function is $n(d)$.

Throughout the paper, we use three important equations in the derivations:
1. $\frac{\partial N(d)}{\partial d}=n(d)=\frac{1}{\sqrt{2 \pi}} \exp \left(-\frac{d^{2}}{2}\right)$
2. $\frac{\partial n(d)}{\partial d}=-d \times n(d)$
3. $S_{0} n\left(d_{1}\right)=X e^{-r t} n\left(d_{2}\right)$ 
Algebraically, the option theta is the partial derivative of the option's value with respect to time to maturity. The follow equations provide close-form expressions of Black-Scholes call and put option thetas, $\theta_{C}$ and $\theta_{P}$ :

$$
\begin{aligned}
C & =S_{0} N\left(d_{1}\right)-X e^{-r t} N\left(d_{2}\right) \\
\theta_{C} & =\frac{\partial C}{\partial t}=S_{0} n\left(d_{1}\right) \frac{\partial d_{1}}{\partial t}-X e^{-r t} n\left(d_{2}\right) \frac{\partial d_{2}}{\partial t}+r X e^{-r t} N\left(d_{2}\right) \\
& =S_{0} n\left(d_{1}\right) \frac{\partial\left(d_{1}-d_{2}\right)}{\partial t}+r X e^{-r t} N\left(d_{2}\right) \\
& =S_{0} n\left(d_{1}\right) \frac{\partial(\sigma \sqrt{t})}{\partial t}+r X e^{-r t} N\left(d_{2}\right) \\
\theta_{C} & =\frac{1}{2 \sqrt{t}} S_{0} n\left(d_{1}\right) \sigma+r X e^{-r t} N\left(d_{2}\right)>0
\end{aligned}
$$

The put option theta can be derived from Eq. 1, using put-call parity for European-style options $\left(S_{0}+P=C+X e^{-r t}\right)$ :

$$
\begin{gathered}
P=C+X e^{-r t}-S_{0} \\
\theta_{P}=\frac{\partial P}{\partial t}=\frac{\partial\left(C+X e^{-r t}-S_{0}\right)}{\partial t}=\theta_{C}-r X e^{-r t} \\
\theta_{P}=\frac{1}{2 \sqrt{t}} S_{0} n\left(d_{1}\right) \sigma-r X e^{-r t}\left(1-N\left(d_{2}\right)\right)
\end{gathered}
$$

From Eq. 2 we have:

$$
\theta_{C}-\theta_{P}=r X e^{-r t}
$$

Note that Eqs. 1 and 3 depend on the Black-Scholes option pricing formula. However, Eq. 4 holds in general regardless validity of the Black-Scholes model assumptions. It can be easily derived from the put-call parity relation of Europeanstyle options, which is based on no-arbitrage conditions.

\section{Upper and lower bounds on thetas}

In this section, we derive the upper and lower bounds on call and put option thetas as functions of underlying asset value, $S_{0}$. The boundary conditions provide the maximum and minimum rates at which the option's time premium dissipates over time.

Equation 1 shows that the call option theta is a sum of two strictly positive terms. As a result, the call option theta is always positive. The time premium of a European-style call option is positively related to its time to maturity. As time to maturity decreases, the call option's time premium always decreases. Holding all 
other parameters constant, as the underlying asset value goes to positive infinity, the call option theta approaches $r X e^{-r t}$. As the underlying asset value goes to zero, the call option theta approaches zero, which is the lower bound of the call option theta.

From Eq. 3, we can see that the Black-Scholes put option theta approaches zero as the underlying asset value goes to positive infinity, and that as the underlying asset value goes to zero, the theta approaches $-r X e^{-r t}$, which is the lower bound of the put option theta. Also note that the put option theta is the difference between two positive terms, and consequently, the difference can be either positive or negative.

Figure 1 provides a graphical presentation of Black-Scholes option thetas as functions of underlying asset value. The parameter values used in the Black-Scholes option-pricing model in Fig. 1 are $X=\$ 100, t=2$ months $=0.1667$ years, $r=5 \%$, and $\sigma=0.40$. The figure demonstrates the following five properties of option thetas:

(1) as $S_{0} \rightarrow 0, \theta_{C} \rightarrow 0$,

(2) as $S_{0} \rightarrow+\infty, \theta_{C} \rightarrow r X e^{-r t}=4.96$,

(3) as $S_{0} \rightarrow 0, \theta_{P} \rightarrow-r X e^{-r t}=-4.96$, and

(4) as $S_{0} \rightarrow+\infty, \theta_{P} \rightarrow 0$.

(5) Although not visually obvious from the graph because of the changes in slope, the difference between $\theta_{C}$ and $\theta_{P}$ is constant with respect to the value of the underlying asset, i.e., for the given values of $t, r$, and, $X, \theta_{C}-\theta_{P}=r X e^{-r t}=4.96$, $\forall S_{0} \in R^{+}$. This result can be seen directly in Eq. 4, which shows that the difference, $r X e^{-r t}$, does not depend on the underlying asset's value. Of course, the difference does depend on $t, r$, and $X$. Therefore, note that the difference increases with the decline in $t$ as the options approach expiration. Also, the difference would increase if there were an increase in either the risk-free interest rate (as long as $1-r t>0$ ) or the exercise price.

In Fig. 1, note that there is a critical point where the put option theta curve intersects the $x$-axis. Below that critical value of the underlying asset, the put option theta is negative. Therefore, a sufficiently low underlying asset value causes the rate of time premium decay on a European-style put option to be negative. In such a case, a decrease in the time to maturity actually causes an increase in the time premium and value of the put option. Above the critical value, the put option theta is positive, and just as it does with a call option, a decrease in the time to maturity causes a decrease in the time premium and value of the put option.

\section{Maximum theta and underlying asset value}

Traders often use options to hedge or speculate. The decay of an option's time premium is an important consideration in the cost of such trading strategies. In this section, we examine call and put option thetas as a function of underlying asset

\footnotetext{
${ }^{1}$ As $S_{0}$ approaches to positive infinity, $n\left(d_{1}\right)$ approaches to zero at a faster rate than $S_{0}$ grows. Consequently the first product term in Eq. 1 approaches to zero. $N\left(d_{2}\right)$ approaches to 1 as $S_{0}$ approaches to positive infinity. As a result, the whole expression in Eq. 1 approaches to $r X e^{-r t}$.
} 
Black-Scholes Call and Put Option Thetas As a Function of Underlying Asset Value

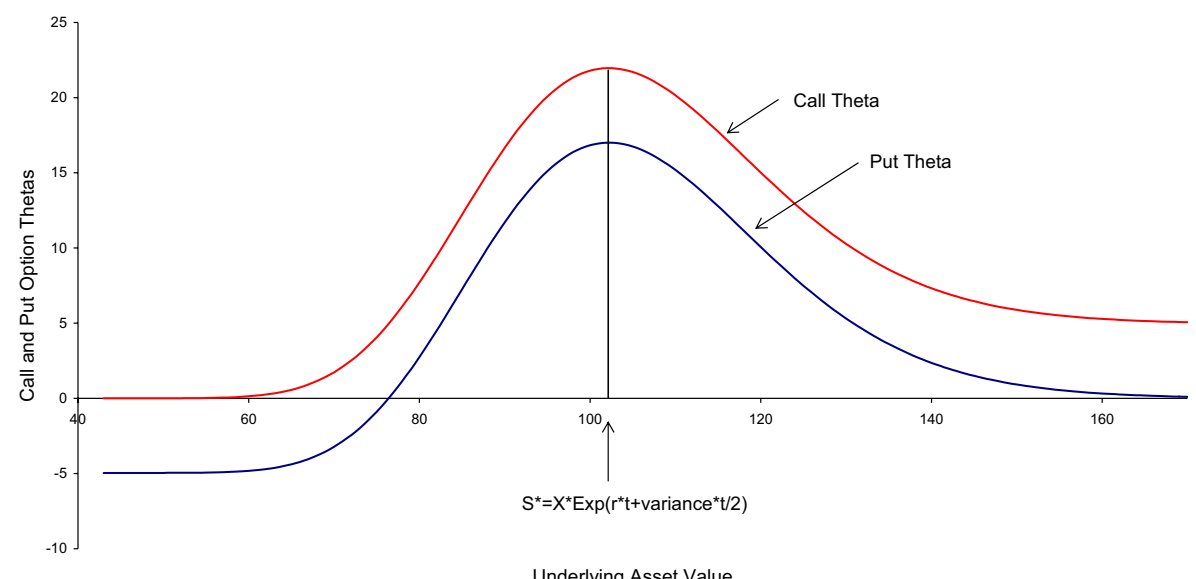

Underlying Asset Value

Fig. 1 Black-Scholes call and put option thetas as a function of underlying asset value. This figure plots Black-Scholes call and put option thetas as a function of underlying asset value. Parameters in the BlackScholes option-pricing model are $X=\$ 100, t=2$ months $=2 / 12$ years, $r=5 \%$, and $\sigma=0.40$. The figure demonstrates the following six properties of option thetas: (1) as $S_{0} \rightarrow 0, \theta_{C} \longrightarrow 0$, (2) as $S_{0} \rightarrow+\infty, \theta_{C} \rightarrow r X e^{-r t}$ $=4.96$, (3) as $S_{0} \rightarrow 0, \theta_{P} \rightarrow-r X e^{-r t}=-4.96$, (4) as $S_{0} \rightarrow+\infty, \theta_{P} \rightarrow 0$, and (5) $\arg \max \left(\theta_{C}\right)=\arg \max \left(\theta_{P}\right)=S^{*}=X$ $\exp \left(r t+\sigma^{2} t / 2\right)=102.19$. Further, even though it may not be visually obvious, the differences between the call option theta and put option theta stay at a constant across all moneyness, i.e., (6) $\theta_{C}-\theta_{P}=r X e^{-r t}=4.96$, $\forall S_{0} \in \mathrm{R}^{+}$

value. In particular, we determine the underlying asset value at which option thetas reach their maximums. Recall from Eq. 4 that the difference between the call and put option thetas is a constant with respect to the value of the underlying asset, so that the sensitivities of call and put option thetas with respect to underlying asset value are identical. Therefore, call and put option thetas reach their maximum at the same underlying asset value. This statement holds true independent from Black-Scholes assumptions because Eq. 4 can be implied by European-style option put-call parity relation.

It is well known that, with all else equal, an option's time premium achieves its maximum value when the value of the underlying asset equals the exercise price (Merton 1973; Smith 1976). From this result, intuition might lead to a belief that option thetas would also achieve their maximum at this same point. However, we show that this is not the case. The maximum option theta is reached when the partial derivative of theta with respect to underlying asset value equals zero. In Appendix A, we derive the underlying asset value that maximizes European-style call and put options thetas by setting $\frac{\partial \theta_{P}}{\partial S_{0}}=\frac{\partial \theta_{C}}{\partial S_{0}}=0$. Appendix A shows that option thetas actually achieve their maximum value when the underlying asset is somewhat above the exercise price:

$$
\arg \max \left(\theta_{C}\right)=\arg \max \left(\theta_{P}\right)=S^{*}=X \exp \left(r t+\sigma^{2} t / 2\right)
$$

Therefore, option thetas for both call and put options are maximized when the underlying asset value exceeds the exercise price by a factor of $\exp \left(r t+\sigma^{2} t / 2\right)-1$. 
This point is in-the-money for the call option, and out-of-the-money for the put option. For example, with $r=5 \%, t=2 / 12$, and $\sigma=0.40$, this factor equals

$$
\exp \left(r t+\sigma^{2} t / 2\right)-1=\exp \left(0.05 \times 2 / 12+0.40^{2} \times 2 / 12 / 2\right)-1=0.0219
$$

Therefore, with these parameter values, option thetas achieve their maximum values when the value of the underlying asset is $2.19 \%$ above the exercise price. With $X=\$ 100$, the maximum theta values would occur at an underlying asset value of $\$ 102.19$. Figure 1 illustrates this relation by graphing option theta values as a function of underlying asset value for these values of the risk-free interest rate, time to maturity, and underlying asset return volatility.

From Eq. 5, we can see that the underlying asset value at which the option thetas reach their maximum decreases with the decline in $t$ as the options approach expiration. It also shows that the underlying asset value that maximizes the option thetas increases with increases in the other variables, the risk-free interest rate, the volatility of the returns of the underlying asset, the exercise price.

\section{Non-monotonicity of theta as a function of sigma}

Option value, and more specifically, an option's time premium, is known to be a monotonic function of the underlying asset's return volatility; the higher the volatility, the larger the time premium, and consequently, the higher the value of the option. Again, intuition might lead one to speculate that the same would hold for option thetas, and again, intuition would lead us astray.

In this section, we show that option thetas are not monotonic functions of return volatility. We first note that the sensitivity of the call option theta with respect to return volatility is exactly the same as it is for that of the put option. Once again, this result is clear from Eq. 4, and parallels the sensitivity to underlying asset value. Appendix B derives the partial derivative of the option thetas with respect to underlying asset return volatility. Specifically, Appendix B shows that:

$$
\frac{\partial \theta_{C}}{\partial \sigma}=\frac{\partial \theta_{P}}{\partial \sigma}=\frac{S_{0} n\left(d_{1}\right)}{2 t^{1.5} \sigma^{2}}\left[\left[\ln \left(S_{0} / X\right)\right]^{2}-r^{2} t^{2}-0.25 \sigma^{4} t^{2}+\sigma^{2} t-r \sigma^{2} t^{2}\right]
$$

The partial derivative can be positive or negative, which proves that the option thetas are not monotonic in return volatility.

Figure 2 plots the sensitivity of Black-Scholes call and put option thetas as a function of sigma, the return volatility of the underlying stock. This sensitivity is the partial derivative of the call and put option theta with respect to sigma. This sensitivity changes dramatically as sigma approaches zero from the positive side. Therefore, to provide a clearer figure, we transform the horizontal axis from sigma to the logarithm of sigma. For example, 0.0 on the horizontal axis represents $\ln (\operatorname{sigma})=0.0$, or sigma $=\exp (0.0)=100 \%$ of annualized underlying asset return volatility. Similarly, -2.0 on the horizontal axis represents $\ln (\operatorname{sigma})=-2.0$, or $\operatorname{sigma}=\exp (-2.0)=$ $13.53 \%$ of annualized underlying asset return volatility. Our transformation stretches the curve horizontally as sigma approaches zero. Parameters in the Black-Scholes option-pricing model are $S_{0}=\$ 100, X=\$ 100, t=6$ months $=0.5$ years, and $r=5 \%$. As 


\section{Black-Scholes Option Theta Sensitivity with Respect to Sigma}

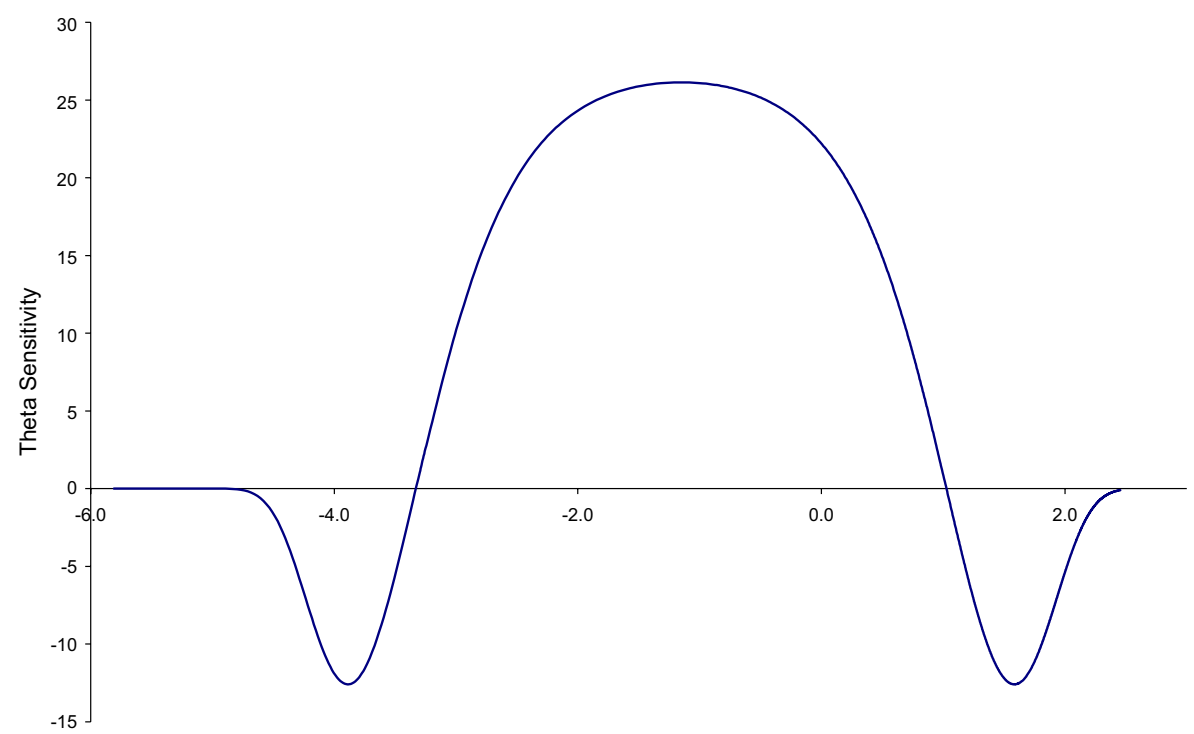

Log Sigma

Fig. 2 Black-Scholes call and put option theta sensitivity with respect to changes in sigma. This figure plots the sensitivity of Black-Scholes call and put option thetas as a function of sigma, the return volatility of the value of the underlying asset. The vertical axis is the partial derivative of both call and put option thetas with respect to sigma. The horizontal axis is the logarithm of sigma. For example, -2.0 on the horizontal axis represents $\ln (\operatorname{sigma})=-2.0$, or $\operatorname{sigma}=\exp (-2.0)=13.53 \%$ of annualized security return volatility. Parameters in the Black-Scholes option-pricing model are $S_{0}=\$ 100, X=\$ 100, t=6$ months $=$ $6 / 12$ years, and $r=5 \%$. As shown mathematically in Appendix B, the figure confirms that Black-Scholes option thetas are not monotonic in sigma. Theta sensitivity becomes negative when sigma is sufficiently close to zero or sufficiently large. Note that the sensitivity of call option theta with respect to changes in sigma is the same as that of a put option theta

is shown mathematically in Appendix B, the figure confirms that Black-Scholes option thetas are not monotonic in sigma. The sensitivity of option thetas with respect to sigma becomes negative when sigma is sufficiently close to zero or sufficiently large. However, for most commonly seen return volatilities of $20 \%$ to $200 \%$ ( -1.6 to 0.7 on the log volatility scale on the horizontal axis), Fig. 2 shows that the sensitivity of theta to sigma is positive.

\section{Non-monotonicity of theta as a function of time to maturity}

We also study the behavior of option thetas as a function of an option's remaining time to maturity. It is well known that option value generally declines as time to maturity dissipates. In particular, the value of a European-style call option declines monotonically as time to maturity dissipates. However, there are parameter values for which the value of a European-style put option can actually increase as time to maturity dissipates, so that a put option's time premium is not strictly monotonic in time to maturity. 
In this section, we show that call and put option thetas can either increase or decrease as the option's time to maturity dissipates. Therefore, option thetas are not monotonic functions of time to maturity. Note that, unlike the situations with underlying asset value and sigma, the sensitivities of the call and put option thetas with respect to time to maturity are not the same.

Figure 3 plots Black-Scholes call option thetas as a function of the option's time to maturity. There are three curves in the figure. Parameters in the Black-Scholes option-pricing model are $X=\$ 100, r=5 \%$, and $\sigma=0.40$. The top curve plots at-themoney call option thetas, where $S_{0}=\$ 100$. The middle curve plots in-the-money call option thetas, where $S_{0}=\$ 120$. The bottom curve plots out-of-the-money call option thetas, where $S_{0}=\$ 80$. Note that near-the-money option thetas behave similarly to atthe-money options thetas. As the option's time to maturity dissipates, away-fromthe-money call option thetas decrease, but at-the-money call option thetas increase. The directions of the three curves match intuition. We omit put option thetas from the figure because one can easily use Eq. 4 to compute put option thetas. Note that as a put option's time to maturity approaches zero, its theta approaches the call option theta minus $r X$, i.e., as $t \rightarrow 0, \theta_{P} \rightarrow \theta_{C}-r X$.

\section{Call Option Thetas As a Function of Time to Maturity}

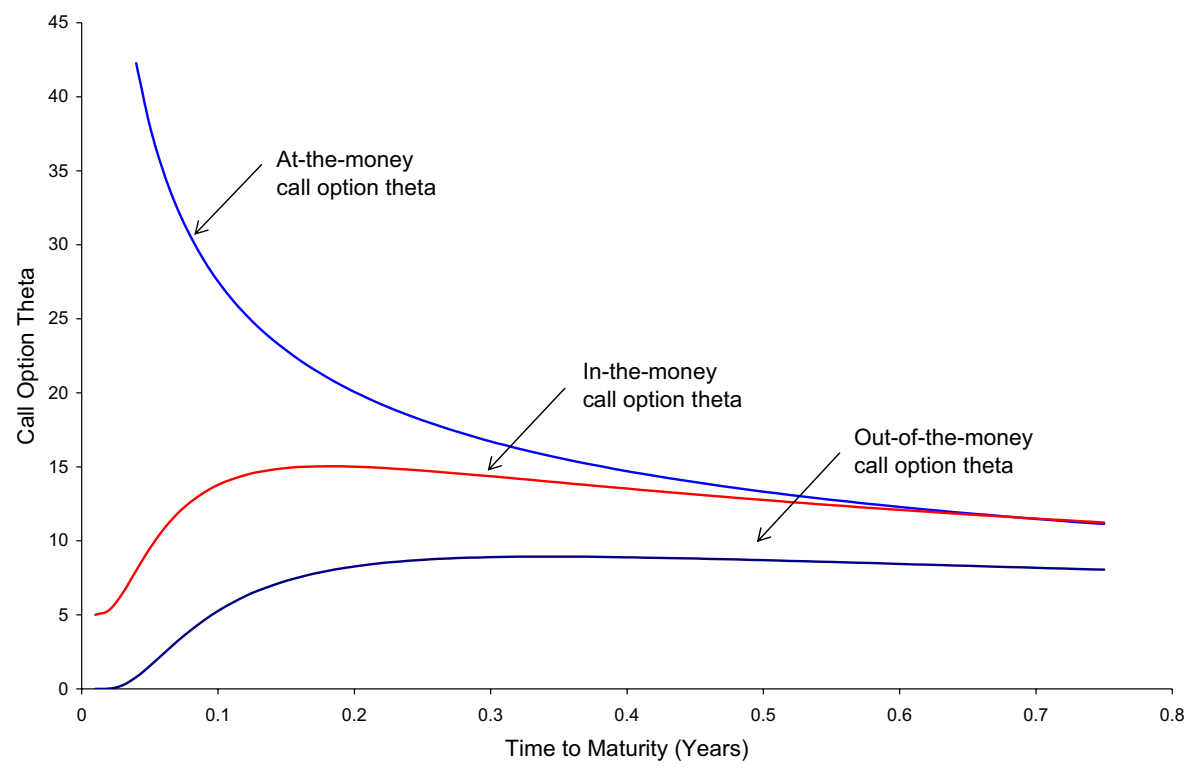

Fig. 3 Black-Scholes call option thetas as a function of time to maturity. This figure plots Black-Scholes call option thetas as a function of option's time to maturity and moneyness. Parameters in the BlackScholes option-pricing model are $X=\$ 100, r=5 \%$, and $\sigma=0.40$. There are three curves in the figure. The top curve plots at-the-money call option thetas, where $S_{0}=\$ 100$. The middle curve plots in-the-money call option thetas, where $S_{0}=\$ 120$. The bottom curve plots out-of-the-money call option thetas, where $S_{0}=\$ 80$. As option's time to maturity dissipates, away-from-the-money call option thetas decrease, but at-themoney call option thetas increase 


\section{Non-monotonicity of theta as a function of the risk-free interest rate}

Finally, we examine Black-Scholes option thetas as a function of the risk-free interest rate. In Appendix D we derive the sensitivity of Black-Scholes call and put option thetas with respect to changes in the risk-free interest rate. The partial derivatives of thetas are not always positive for both call and put options. Consequently, the Black-Scholes option thetas are not monotonic functions of the risk-free interest rate.

Figure 4 plots the partial derivative of Black-Scholes call and put option thetas with respect to the risk-free interest rate. Parameters in the Black-Scholes optionpricing model are $S_{0}=\$ 80, X=\$ 100, t=6$ months, and $\sigma=0.70$. The top curve plots call option theta sensitivity and the bottom curve is the put option theta sensitivity. The call option theta sensitivity is positive for most parameter values. However, for very large values of sigma (not shown in Fig. 4), the call option curve can go negative, showing numerically that the Black-Scholes call option theta is not monotonic functions of the risk-free interest rate. Non-monotonic behavior of theta occurs only using extreme values of sigma.

Black-Scholes Option Theta Sensitivity to Risk-Free Interest Rate

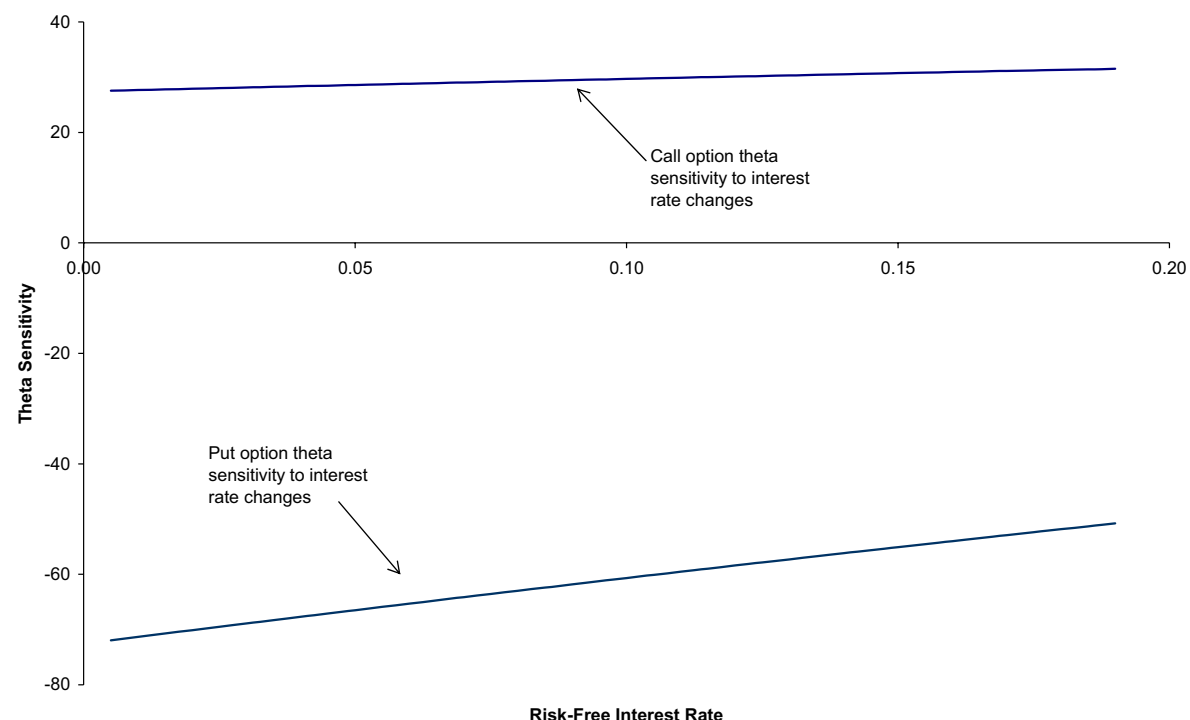

Fig. 4 Black-Scholes call and put option theta sensitivity with respect to changes in risk-free interest rate. This figure plots the sensitivity of Black-Scholes call and put option thetas as a function of risk-free interest rate. The vertical axis is the partial derivative of both call and put option thetas with respect to $r$. The horizontal axis is the risk-free interest rate. Parameters in the Black-Scholes option-pricing model are $S_{0}=\$ 80, X=\$ 100, t=6$ months $=6 / 12$ years, and sigma $=0.70$. The exact mathematical expressions of partial derivatives are given in Appendix D. Note that the sensitivity of call option theta to interest rate changes tends to be positive for most parameter settings, but the sensitivity can become negative (not shown in this figure) for very large values of sigma 


\section{Trading implications}

Hedgers and speculators who take option positions can make use of our results. Figure 1 and Eq. 5 show that somewhat in-the-money call options and somewhat out-of-the-money put options have the highest theta. All else equal, a trader taking a long position in such options will tend to suffer the most loss from time premium decay. Consequently, in taking a long position, a trader may want to consider using farther away-from-the-money options to minimize the amount lost to time premium decay. In the mirror-image case, a trader taking a short position will tend to reap the largest gain from time premium decay if the value of the underlying asset is somewhat above the exercise price. Consequently, in taking a short position, a trader may want to consider using options with an exercise price that is somewhat below the value of the underlying asset to maximize the amount gained from time premium decay.

A trader engaging in a bull call option spread buys a call option with a low exercise price and sells a call option with a higher exercise price to anticipate potential upward movement in the underlying asset's value prior to the option's expiration. If the trader uses call options that are both in-the-money, then Fig. 1 implies that the call option with the higher exercise price has a higher theta than the call option with a lower exercise price. As a result, the trader gains more from time premium decay on the short call option with the higher exercise price than the trader loses from time premium decay on the long call option with the lower exercise price. The trader captures a net gain from time premium decay in the bull spread. However, taking a bull call option spread using out-of-the-money call options reverses the result. Figure 1 implies that a bull spread using out-of-the-money call options result in a net loss from time premium decay.

A calendar spread (also known as a time, or horizontal, spread) involves buying an option and selling an otherwise identical option with a different time to maturity. Most trading strategies related to calendar spreads are "time-premium plays."

Figure 3 offers insights into how to profit from a call option calendar spread. If the options are at-the-money, the trader should sell the shorter-time-to-maturity option and buy the longer-time-to-maturity option because the at-the-money option theta line in Fig. 3 shows that shorter-time-to-maturity options lose time premium at a higher rate than longer-time-to-maturity options. If the call options are away-fromthe-money, then the bottom two lines in Fig. 3 suggest an opposite strategy, i.e., buy the shorter-time-to-maturity option and sell the longer-time-to-maturity option, because longer-time-to-maturity options lose time premium at a higher rate than shorter-time-to-maturity options. Consequently, a trader should buy an option with little time to maturity and sell a longer-time-to-maturity option to construct a profitable calendar spread.

We have provided two specific examples of trading strategies where time premium decay might be managed to an advantage. There are, of course, other strategies to which the results here can also be generalized, such as straddles, straps, strips, butterfly spreads, ratio spreads, among others. A more complete understanding of option thetas can help market participants to formulate better option trading strategies. 


\section{Conclusion}

This paper examines call and put option thetas within the Black-Scholes optionpricing framework. Specifically, we address five issues. First, Black-Scholes call and put option thetas are bounded. We provide their upper and lower bounds and discuss their relation. Second, we derive the critical underlying asset value that maximizes call and put option thetas. Third, we demonstrate that Black-Scholes option thetas are not monotonic functions of the return volatility of the underlying asset. Fourth, we show how remaining time to maturity affects option thetas. Fifth, we derive the relation between option thetas and the risk-free interest rate.

Applying the results from this paper, we discuss important trading and hedging implications associated with option thetas. We show that a bull option spread with in-the-money call options is more favorable with the short (long) position in the option that has the higher (lower) exercise price. We also show that a call option calendar spread is more favorable with the short (long) position in the option that has the shorter (longer) time to maturity. Parallel conclusions hold for many other spread strategies, such as butterfly spreads and ratio spreads.

Our findings have particular relevance for trading and hedging strategies in risk management that are affected by the decay in an option's time premium.

Acknowledgment We thank Gordon Alexander for helpful comments.

\section{Appendix A}

This appendix derives the underlying asset value that maximizes a European-style call and put options' thetas. Specifically, we prove the following equation based on the Black-Scholes option pricing framework:

$$
\arg \max \left(\theta_{C}\right)=\arg \max \left(\theta_{P}\right)=S^{*}=X \exp \left(r t+\sigma^{2} t / 2\right) .
$$

The derivation is based on the first-order condition. We compute the partial derivative of the option's theta, set it to zero, and solve for the critical value of the underlying asset.

$$
\begin{aligned}
\frac{\partial \theta_{P}}{\partial S_{0}} & =\frac{\partial\left(\theta_{C}-r X e^{-r t}\right)}{\partial S_{0}}=\frac{\partial \theta_{C}}{\partial S_{0}}=\frac{\partial\left[\frac{1}{2 \sqrt{t}} S_{0} n\left(d_{1}\right) \sigma+r X e^{-r t} N\left(d_{2}\right)\right]}{\partial S_{0}} \\
& =\frac{1}{2 \sqrt{t}} n\left(d_{1}\right) \sigma+\frac{1}{2 \sqrt{t}} S_{0} n\left(d_{1}\right) \sigma\left(-d_{1}\right) \frac{1}{S_{0} \sigma \sqrt{t}}+r X e^{-r t} n\left(d_{2}\right) \frac{1}{S_{0} \sigma \sqrt{t}} \\
& =\frac{1}{2 \sqrt{t}} n\left(d_{1}\right) \sigma-\frac{1}{2 t} n\left(d_{1}\right) d_{1}+r S_{0} n\left(d_{1}\right) \frac{1}{S_{0} \sigma \sqrt{t}} \\
& =\frac{1}{2 \sqrt{t}} n\left(d_{1}\right) \sigma-\frac{1}{2 t} n\left(d_{1}\right) d_{1}+r n\left(d_{1}\right) \frac{1}{\sigma \sqrt{t}} \\
& =n\left(d_{1}\right)\left[\frac{1}{2 \sqrt{t}} \sigma-\frac{1}{2 t} d_{1}+r \frac{1}{\sigma \sqrt{t}}\right]
\end{aligned}
$$




$$
\begin{aligned}
& =n\left(d_{1}\right) \frac{\sigma^{2} \sqrt{t}-d_{1} \sigma+2 r \sqrt{t}}{2 \sigma t} \\
& =n\left(d_{1}\right) \frac{\sigma^{2} t-\left(\ln \left(S_{0} / X\right)+\left(r+\sigma^{2} / 2\right) t\right)+2 r t}{2 \sigma t \sqrt{t}} \\
& =-n\left(d_{1}\right) \frac{\ln \left(S_{0} / X\right)-r t-\sigma^{2} t / 2}{2 \sigma t \sqrt{t}}
\end{aligned}
$$

Setting $\frac{\partial \theta_{P}}{\partial S_{0}}=\frac{\partial \theta_{C}}{\partial S_{0}}=0$, we obtain $S_{0}^{*}=X e^{\left(r t+\sigma^{2} t / 2\right)}=X \times \exp \left(r t+\sigma^{2} t / 2\right)$.

\section{Appendix B}

This appendix shows that option thetas are not monotonic functions of sigma, the return volatility of the underlying asset. We derive the partial derivative of thetas with respect to sigma and show that the partial derivative can be either positive or negative, which proves that the option thetas are not monotonic in sigma. The following derivation uses two equations $\frac{\partial d_{1}}{\partial \sigma}=\frac{-d_{2}}{\sigma}$ and $\frac{\partial d_{2}}{\partial \sigma}=\frac{-d_{1}}{\sigma}$.

$$
\begin{aligned}
& \frac{\partial \theta_{P}}{\partial \sigma}=\frac{\partial\left(\theta_{C}-r X e^{-r t}\right)}{\partial \sigma}=\frac{\partial \theta_{C}}{\partial \sigma}=\frac{\partial\left[\frac{1}{2 \sqrt{t}} S_{0} n\left(d_{1}\right) \sigma+r X e^{-r t} N\left(d_{2}\right)\right]}{\partial \sigma} \\
& =\frac{1}{2 \sqrt{t}} S_{0} n\left(d_{1}\right)+\frac{1}{2 \sqrt{t}} \sigma S_{0} n\left(d_{1}\right)\left(-d_{1}\right) \frac{\partial d_{1}}{\partial \sigma}+r X e^{-r t} n\left(d_{2}\right) \frac{\partial d_{2}}{\partial \sigma} \\
& =\frac{1}{2 \sqrt{t}} S_{0} n\left(d_{1}\right)+\frac{1}{2 \sqrt{t}} \sigma S_{0} n\left(d_{1}\right)\left(-d_{1}\right) \frac{\partial d_{1}}{\partial \sigma}+r S_{0} n\left(d_{1}\right)\left(\frac{\partial d_{2}}{\partial \sigma}\right) \\
& =\frac{1}{2 \sqrt{t}} S_{0} n\left(d_{1}\right)+\frac{1}{2 \sqrt{t}} \sigma S_{0} n\left(d_{1}\right)\left(-d_{1}\right) \frac{-d_{2}}{\sigma}+r S_{0} n\left(d_{1}\right)\left(\frac{-d_{1}}{\sigma}\right) \\
& =\frac{S_{0} n\left(d_{1}\right)}{2 \sqrt{t} \sigma}\left[\sigma+d_{1} d_{2} \sigma-2 \sqrt{t} r d_{1}\right] \\
& =\frac{S_{0} n\left(d_{1}\right)}{2 \sqrt{t} \sigma}\left[\sigma+\frac{\ln \left[S_{0} /\left(X e^{-r t}\right)\right]+\frac{1}{2} \sigma^{2} t}{\sigma \sqrt{t}} \frac{\ln \left[S_{0} /\left(X e^{-r t}\right)\right]-\frac{1}{2} \sigma^{2} t}{\sigma \sqrt{t}} \sigma\right. \\
& \left.-2 \sqrt{t} \frac{\ln \left[S_{0} /\left(X e^{-r t}\right)\right]+\frac{1}{2} \sigma^{2} t}{\sigma \sqrt{t}}\right] \\
& =\frac{S_{0} n\left(d_{1}\right)}{2 \sqrt{t} \sigma}\left[\sigma+\frac{\left\{\ln \left[S_{0} /\left(X e^{-r t}\right)\right]\right\}^{2}-0.25 \sigma^{4} t^{2}}{\sigma t}-\frac{2 r}{\sigma}\left[\ln \left[S_{0} /\left(X e^{-r t}\right)\right]+\frac{1}{2} \sigma^{2} t\right]\right] \\
& =\frac{S_{0} n\left(d_{1}\right)}{2 t^{1.5} \sigma^{2}}\left[\left\{\ln \left[S_{0} /\left(X e^{-r t}\right)\right]\right\}^{2}-2 r t\left\{\ln \left[S_{0} /\left(X e^{-r t}\right)\right]\right\}-0.25 \sigma^{4} t^{2}+\sigma^{2} t-r \sigma^{2} t^{2}\right] \\
& =\frac{S_{0} n\left(d_{1}\right)}{2 t^{1.5} \sigma^{2}}\left[\left[\ln \left(S_{0} / X\right)\right]^{2}-r^{2} t^{2}-0.25 \sigma^{4} t^{2}+\sigma^{2} t-r \sigma^{2} t^{2}\right]
\end{aligned}
$$

The term, " $-r^{2} t^{2}-0.25 \sigma^{4} t^{2}+\sigma^{2} t-r \sigma^{2} t^{2 "}$ can be negative, as $\sigma$ approaches zero or positive infinity. It implies that the whole expression $\frac{\partial \theta_{P}}{\partial \sigma}=\frac{\partial \theta_{C}}{\partial \sigma}$ can be either positive 
or negative, i.e., call and put option thetas are not monotonic functions in the return volatility of the underlying asset.

\section{Appendix C}

This appendix derives the expression of the sensitivity of option theta to changes in the option's time to maturity. The expression of the partial derivative of theta with respect to time to maturity does not allow us to solve for the critical remaining life that will maximize theta. Numerical methods are needed to solve this issue.

$$
\begin{aligned}
& \frac{\partial \theta_{C}}{\partial t}=\frac{\partial\left[\frac{1}{2 \sqrt{t}} S_{0} n\left(d_{1}\right) \sigma+r X e^{-r t} N\left(d_{2}\right)\right]}{\partial t} \\
& =-\frac{1}{4 t \sqrt{t}} S_{0} n\left(d_{1}\right) \sigma+\frac{1}{2 \sqrt{t}} S_{0} n\left(d_{1}\right) \sigma\left(-d_{1}\right) \frac{\partial d_{1}}{\partial t}-r^{2} X e^{-r t} N\left(d_{2}\right) \\
& +r X e^{-r t} n\left(d_{2}\right) \frac{\partial d_{2}}{\partial t} \\
& =-\frac{1}{4 t \sqrt{t}} S_{0} n\left(d_{1}\right) \sigma+\frac{1}{2 \sqrt{t}} S_{0} n\left(d_{1}\right) \sigma\left(-d_{1}\right)\left[\left(r+\frac{\sigma^{2}}{2}\right) \frac{1}{\sigma \sqrt{t}}-\frac{d_{1}}{2 t}\right] \\
& -r^{2} X e^{-r t} N\left(d_{2}\right)+r X e^{-r t} n\left(d_{2}\right)\left[\frac{r}{\sigma \sqrt{t}}-\frac{d_{1}}{2 t}\right] \\
& =-\frac{S_{0} n\left(d_{1}\right)}{4 \sigma t \sqrt{t}}\left[\sigma^{2}+d_{1} \sigma 2 t\left[\left(r+\frac{\sigma^{2}}{2}\right) \frac{1}{\sigma \sqrt{t}}-\frac{d_{1}}{2 t}\right]-4 \sqrt{t} r \sigma\left(\frac{r}{\sigma \sqrt{t}}-\frac{d_{1}}{2 t}\right)\right] \\
& -r^{2} X e^{-r t} N\left(d_{2}\right) \\
& =-\frac{S_{0} n\left(d_{1}\right)}{4 \sigma t \sqrt{t}}\left[\sigma^{2}+d_{1} \sigma 2 \sqrt{t} r+d_{1} \sigma^{3} \sqrt{t}-d_{1}^{2} \sigma^{2}-4 t r^{2}+2 \sqrt{t} r \sigma d_{1}\right]-r^{2} X e^{-r t} N\left(d_{2}\right) \\
& =-\frac{S_{0} n\left(d_{1}\right)}{4 \sigma t \sqrt{t}}\left[\sigma^{2}+4 \sqrt{t} r \sigma d_{1}-\sigma^{2} d_{1} d_{2}-4 t r^{2}\right]-r^{2} X e^{-r t} N\left(d_{2}\right) \\
& =\frac{S_{0} n\left(d_{1}\right)}{4 \sigma t \sqrt{t}}\left[\sigma^{2} d_{1} d_{2}-4 \sqrt{t} r \sigma d_{1}+4 t r^{2}-\sigma^{2}\right]-r^{2} X e^{-r t} N\left(d_{2}\right) \\
& \frac{\partial \theta_{P}}{\partial t}=\frac{\partial\left(\theta_{C}-r X e^{-r t}\right)}{\partial t}=\frac{\partial \theta_{C}}{\partial t}+r^{2} X e^{-r t} \\
& =\frac{S_{0} n\left(d_{1}\right)}{4 \sigma t \sqrt{t}}\left[\sigma^{2} d_{1} d_{2}-4 \sqrt{t} r \sigma d_{1}+4 t r^{2}-\sigma^{2}\right]+r^{2} X e^{-r t}\left(1-N\left(d_{2}\right)\right)
\end{aligned}
$$

The above expressions can be either positive or negative in value for various parameter inputs. It shows that call and put option thetas are not monotonic functions in options' remaining time to maturity. 


\section{Appendix D}

This appendix derives the expression of the sensitivity of option theta to changes in the risk-free interest rate. The expression of the partial derivative of theta with respect to the risk-free interest rate does not allow us to sign the expression easily. Numerical methods are needed to resolve this issue.

$$
\begin{aligned}
\frac{\partial \theta_{C}}{\partial r} & =\frac{\partial\left[\frac{1}{2 \sqrt{t}} S_{0} n\left(d_{1}\right) \sigma+r X e^{-r t} N\left(d_{2}\right)\right]}{\partial r} \\
& =\frac{1}{2 \sqrt{t}} S_{0} n\left(d_{1}\right) \sigma\left(-d_{1}\right) \frac{\partial d_{1}}{\partial r}+X e^{-r t} N\left(d_{2}\right)-r t X e^{-r t} N\left(d_{2}\right)+r X e^{-r t} n\left(d_{2}\right) \frac{\partial d_{2}}{\partial r} \\
& =\frac{1}{2 \sqrt{t}} S_{0} n\left(d_{1}\right) \sigma\left(-d_{1}\right) \frac{t}{\sigma \sqrt{t}}+X e^{-r t} N\left(d_{2}\right)-r t X e^{-r t} N\left(d_{2}\right)+r X e^{-r t} n\left(d_{2}\right) \frac{t}{\sigma \sqrt{t}} \\
& =-\frac{1}{2} S_{0} d_{1} n\left(d_{1}\right)+X e^{-r t} N\left(d_{2}\right)-r t X e^{-r t} N\left(d_{2}\right)+r X e^{-r t} n\left(d_{2}\right) \frac{\sqrt{t}}{\sigma} \\
& =X e^{-r t} N\left(d_{2}\right)(1-r t)+X e^{-r t} n\left(d_{2}\right)\left(\frac{r \sqrt{t}}{\sigma}-\frac{d_{1}}{2}\right) \\
\frac{\partial \theta_{P}}{\partial r} & =\frac{\partial\left(\theta_{C}-r X e^{-r t}\right)}{\partial r}=\frac{\partial \theta_{C}}{\partial r}-X e^{-r t}+r t X e^{-r t} \\
& =X e^{-r t} N\left(d_{2}\right)(1-r t)+X e^{-r t} n\left(d_{2}\right)\left(\frac{r \sqrt{t}}{\sigma}-\frac{d_{1}}{2}\right)-X e^{-r t}(1-r t) \\
& =X e^{-r t}(1-r t)\left(N\left(d_{2}\right)-1\right)+X e^{-r t} n\left(d_{2}\right)\left(\frac{r \sqrt{t}}{\sigma}-\frac{d_{1}}{2}\right) \\
& =-r t N\left(-d_{2}\right)(1-r t)+X e^{-r t} n\left(d_{2}\right)\left(\frac{r \sqrt{t}}{\sigma}-\frac{d_{1}}{2}\right)
\end{aligned}
$$

The above expressions can be either positive or negative for various parameter inputs, and demonstrates that call and put option thetas are not monotonic functions of the risk-free interest rate.

\section{References}

Alexander G, Stutzer M (1996) A graphical note on European put thetas. J Futures Mark 16:201-209 Black F (1975) Fact and fantasy in the use of options. Financ Anal J 31:36-72

Black F, Scholes MS (1973) The pricing of options and corporate liabilities. J Polit Econ 81:637-654

Chance DM (1994) Translating the Greek: the real meaning of call option derivatives. Financ Anal J 50(4):43-49

Chung S, Shackleton M (2002) The binomial Black-Scholes model and the Greeks. J Futures Mark 22(2):143-153

Emanuel DC, MacBeth JD (1982) Further results on the constant elasticity of variance call option pricing model. J Financ Quant Anal 18:533-554 
MacBeth JD, Merville LJ (1979) An empirical examination of the Black-Scholes call option pricing model. J Finance 34:1173-1186

Merton RC (1973) Theory of rational option pricing. Bell J Econ Manage Sci 4:141-183

Naib FA (1996) FX option theta and the 'joker' effect. Deriv Q 2(4):56-58

Pelsser A, Vorst T (1994) The binomial model and the Greeks. J Deriv 1(3):45-49

Rubinstein M (1985) Nonparametric tests of alternative option pricing models using all reported trades and quotes on the 30 most active CBOE option classes from August 23, 1976 through August 31, 1978. J Finance 40:455-479

Smith CW Jr (1976) Option pricing: a review. J Financ Econ 3:3-51 
Copyright of Journal of Economics \& Finance is the property of Springer Science \& Business Media B.V. and its content may not be copied or emailed to multiple sites or posted to a listserv without the copyright holder's express written permission. However, users may print, download, or email articles for individual use. 